\title{
Многоканальный измерительный комплекс на базе малогабаритных спектрометров высокого разрешения
}

\author{
Б. Лапшинов, к. т. н. ${ }^{1}$, Н. Тимченко ${ }^{2}$
}

УДК [536.521:535.338.1:535.233]:004.67| ВАК 05.11.07

\begin{abstract}
Исследование современных плазменных технологий требует разработки новых измерительных процессов. Предлагаемый многоканальный измерительный комплекс на базе малогабаритных спектрометров позволяет проводить спектральные исследования как стационарных, так и быстропротекающих процессов с нестационарной температурой.
\end{abstract}

собенности многих высокотемпературных технологических процессов требуют определения как самой температуры, так и спектра излучения нагретого объекта. К таким процессам относятся высокотемпературные процессы микроэлектроники, процессы лазерной и электронно-лучевой обработки материалов и многие другие. Кроме того, исследованию подвергаются как стационарные, так и быстропротекающие технологические процессы с нестационарной температурой, например, при определении температурных полей в выхлопе реактивных двигателей, процессах горения и взрывов, самораспространяющегося высокотемпературного синтеза (CBC). Всё это связано с разработкой новых, динамичных методов диагностики на разных стадиях технологических процессов для определения прочностных характеристик элементов устройств и оборудования, более глубокого понимания физико-химических моделей процессов, а также совершенствования методов математического моделирования. Также следует отметить, что при подобных исследованиях часто требуется определение температуры и спектра излучения не только в наиболее критичной области для данного процесса, но и за ее пределами.

На рынке доступны малогабаритные спектрометры высокого разрешения от различных производителей, таких как Ocean Optics и Avantes, которые предназначены для спектральных диапазонов от ближнего ультрафиолета (УФ) до ближнего инфракрасного (ИК) излучения, в совокупности охватывающих область 1902500 нм. В статье предлагается разработка мобильного измерительного комплекса на базе таких спектрометров

ФГБНУ «Научно-исследовательский институт перспективных материалов и технологий", с.н.с., доцент, Ibaniipmt@mail.ru.

ФГБНу «Научно-исследовательский институт перспективных материалов и технологий", инженер-программист, njtim@mail.ru. для определения температуры и спектра излучения нагретых объектов в различных процессах.

\section{СТРУКТУРА ИЗМЕРИТЕЛЬНОГО КОМПЛЕКСА}

Излучение от исследуемого объекта передается в спектрометр по оптическому волокну, приемный торец которого может быть размещен в необходимом для исследования месте. Стандартный угол сбора информации таким волокном составляет $20^{\circ}$. Используя различные диафрагмы, расположенные на определенном расстоянии от торца волокна, можно значительно уменьшить этот угол и выполнять измерения локальных областей исследуемого объекта.

Спектрометры комплекса работают под управлением программного обеспечения Multi Spectral Pyrometry [1], которое позволяет работать с несколькими (до восьми) спектрометрами одновременно. В процессе работы для каждого спектрометра на управляющем компьютере создаются папки для записи результатов измерений. Программа обеспечивает сбор и обработку числовых данных, полученных со спектрометров Ocean Optics модели HR2000+, выполняет расчет температур в реальном масштабе времени и сохранение исходных спектров в виде текстовых файлов для их последующей обработки и исследования.

Структурная схема измерительного комплекса показана на рис. 1.

Получив спектры теплового излучения объекта в различных точках, можно с помощью метода спектральной пирометрии [2] определить температуру в этих точках. Вычисление электронной температуры плазмы осуществляется путем фиксации спектров плазмы в различных ее областях и определения соотношения интенсивностей отдельных спектральных линий. Кроме того, комплекс может быть применен для определения коэффициентов отражения и пропускания объектов с переменными оптическими характеристиками, а также для других исследований. 


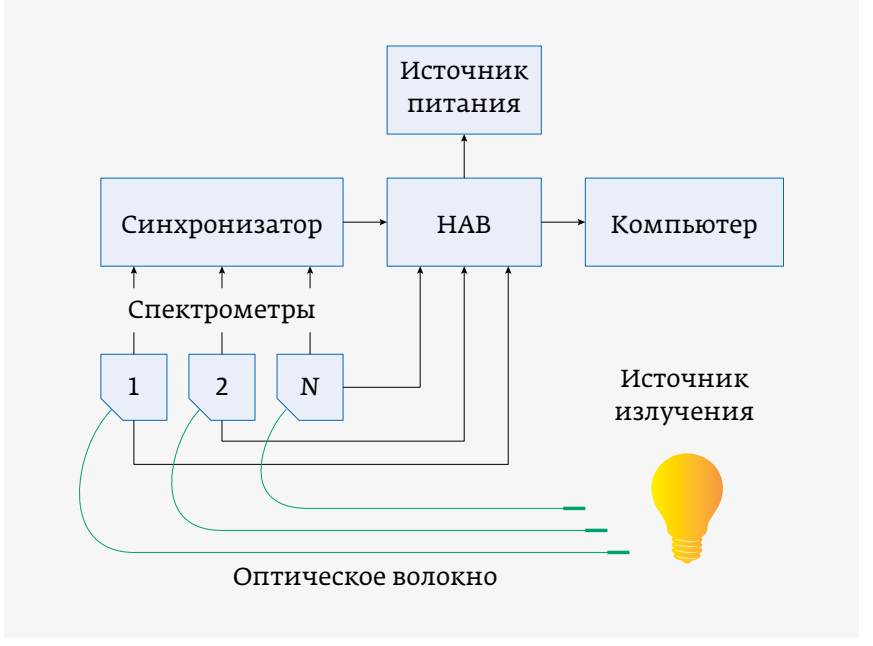

Рис. 1. Структурная схема измерительного комплекса

\section{ИССЛЕДОВАНИЕ ПРОСТРАНСТВЕННЫХ ХАРАКТЕРИСТИК ПРИПОВЕРХНОСТНОЙ ЛАЗЕРНОЙ ПЛАЗМЫ С ИСПОЛЬЗОВАНИЕМ КОМПЛЕКСА}

С применением данного комплекса было проведено исследование пространственных характеристик приповерхностной лазерной плазмы мишеней из меди и алюминия в УФ-и видимой областях спектра.

Приповерхностная лазерная плазма возникает при выполнении многих технологических операций, таких как лазерная резка материалов; лазерная сварка; сверление отверстий; обработка тонких пленок; лазерное импульсное напыление, способное формировать пленки нанометровой толщины; лазерная гравировка и др. Все эти процессы охватывают широкий диапазон плотности потока лазерного излучения Р - от $10^{3}$ до $10^{15}$ Вт / см². Характер процессов, развивающихся при таком взаимодействии, зависит от значения плотности потока на поверхности облучаемого вещества, длины волны лазерного излучения, формы лазерных импульсов, размера области и режима ее облучения, состава и давления окружающего газа, характеристик материала и поверхности мишени [3]. Лазерная плазма является импульсным эмиттером электронов, одно- и многократно ионизованных атомов, нейтральных атомов, молекул с малой и большой энергией. В практических приложениях, особенно при лазерно-плазменном осаждении тонких пленок [4], необходимо иметь информацию о характеристиках эрозионного факела.

В представленных экспериментах лазерная плазма формировалась при действии на мишень импульсного излучения Nd:YAG-лазера ( $\lambda=1,064$ мкм), длительность импульсов составляла порядка 200 мкс, частота следования $\mathrm{F}=3$ кГц. Форма лазерных импульсов близка к треугольной с практически вертикальным передним фронтом

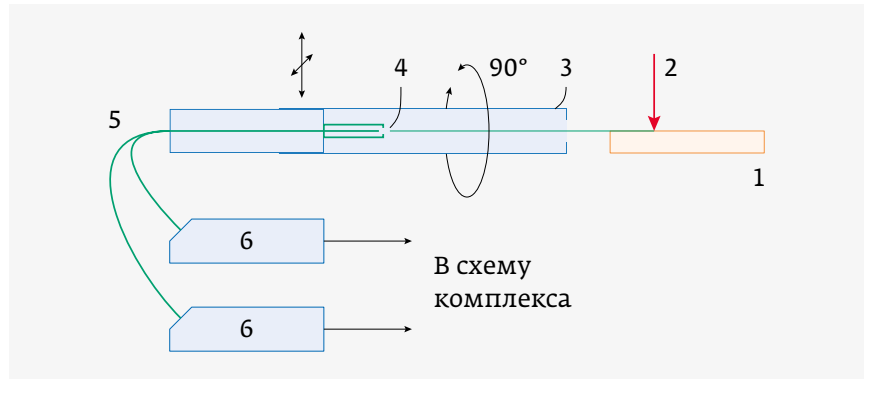

Рис. 2. Схема измерения: 1 - мишень; 2 - луч лазера; 3 - диафрагма; 4 - приемный торец оптического волокна; 5 - сдвоенный оптоволоконный кабель УФ- и видимой области спектра; 6 - спектрометры

и ниспадающим задним. Диаметр лазерного пятна на поверхности мишени $d=30$ мкм. Плотность лазерного излучения при частоте следования импульсов $\mathrm{F}=3$ кГц составляла $\mathrm{P}_{\mathrm{cp}} \approx 2 \cdot 10^{7}$ Вт / см². В качестве мишени использовались массивные образцы меди и алюминия.

В составе измерительного комплекса использовались два спектрометра типа HR2000+ (Ocean Optics, США), имеющие чувствительность в области ближнего ультрафиолета (190-400 нм) и в видимой (400-760 нм) области спектра. Спектральное разрешение приборов не хуже 1 нм (ПЗС-линейка Sony ILX511B, количество пикселей 2048, дифракционная решетка HCl, ширина входной щели 50 мкм). Спектрометры регистрировали абсолютные спектры излучения, то есть количество световой энергии, падающей в единицу времени на единицу площади поверхности (приемный торец оптического волокна). Все измерения проводились в воздушной среде при нормальном атмосферном давлении.

Определение пространственного распределения интенсивностей спектральных линий исследуемых материалов мишеней каждым спектрометром проводилось по схеме, показанной на рис. 2.

Излучение от лазерной плазмы попадало на приемный торец оптического волокна диаметром 0,6 мм через щелевую диафрагму размером 0,15×4 мм. Диафрагма ориентировалась параллельно и перпендикулярно оси лазерного пучка и перемещалась с помощью винтовой пары в горизонтальном и вертикальном направлениях соответственно с шагом 0,05 мм. Величина перемещения определялась с помощью индикатора часового типа с ценой деления 0,01 мм. Используемая геометрия системы позволяла вырезать из плазменного факела область, размер которой примерно соответствовал размеру диафрагмы.

Структура плазменного факела, возникающего при воздействии интенсивного лазерного излучения на поверхность металла, имеет форму, показанную на рис. 3.

В плазменном факеле выделяют три зоны, различающиеся плотностью частиц и характером 


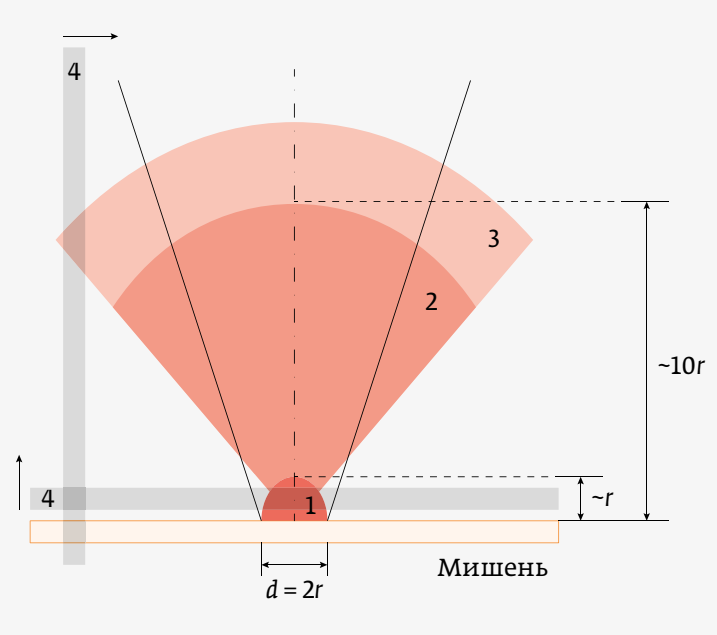

Рис. 3. Структура плазменного факела: 1 ...3 - характерные зоны плазмы; 4 - щелевая диафрагма

газодинамического движения. Зона 1 - плотное горячее ядро факела, поглощающее лазерное излучение и являющееся интенсивным эмиттером ионов. Зона 2 - зона ускорения, в которой происходит переход тепловой энергии плазмы в кинетическую энергию направленного газодинамического движения. В зоне 3 скорость движения частиц плазмы выходит на асимптотическое значение, а тепловая энергия плазмы существенно меньше энергии направленного движения [5].

Таким образом, при каждом новом положении диафрагмы (4) фиксировались интегральные спектры излучения вырезанного ею объема лазерной плазмы, тем самым осуществлялось послойное сканирование плазменного факела в двух направлениях и в двух спектральных областях. В процессе эксперимента пошаговое перемещение диафрагмы производилось от одного крайнего положения с нулевой интенсивностью спектра до другого крайнего положения, то есть через всю область плазменного факела. При воздействии лазерного пучка мишень перемещалась в горизонтальной плоскости с небольшой скоростью, чтобы предотвратить образование глубокого кратера на ее поверхности, что могло сказаться на пространственной форме плазменного факела.

Регистрация спектров излучения меди и алюминия позволила построить зависимость интенсивности некоторых наиболее интенсивных линий от пространственной координаты диафрагмы. Для меди были выбраны как наиболее интенсивные спектральные линии с длинами волн 515,323 и 521,82 нм в видимом диапазоне, а для алюминия - 394,40 и 396,152 нм в УФ-диапазоне [6].

Можно видеть, что в спектрах излучения, содержащего излучение зоны 1 плазменного факела, присутствует составляющая переменной интенсивности, которая объясняется тепловым характером излучения данной зоны. В спектрах излучения вне этой зоны присутствуют только спектральные линии материала мишени.

медь. Типичные спектры меди представлены на рис. 4.

Изменение интенсивностей выбранных спектральных линий меди при перемещении диафрагмы в продольном и поперечном направлениях показано на рис. 5.

Алюминий. Спектры алюминия в УФ-и видимой областях показаны на рис. 6.

Изменение интенсивностей спектральных линий алюминия при перемещении диафрагмы в продольном и поперечном направлениях показано на рис. 7.

Для обоих материалов мишеней при перемещении диафрагмы вверх спектрометр фиксирует излучение выбранных спектральных линий на расстоянии до одного
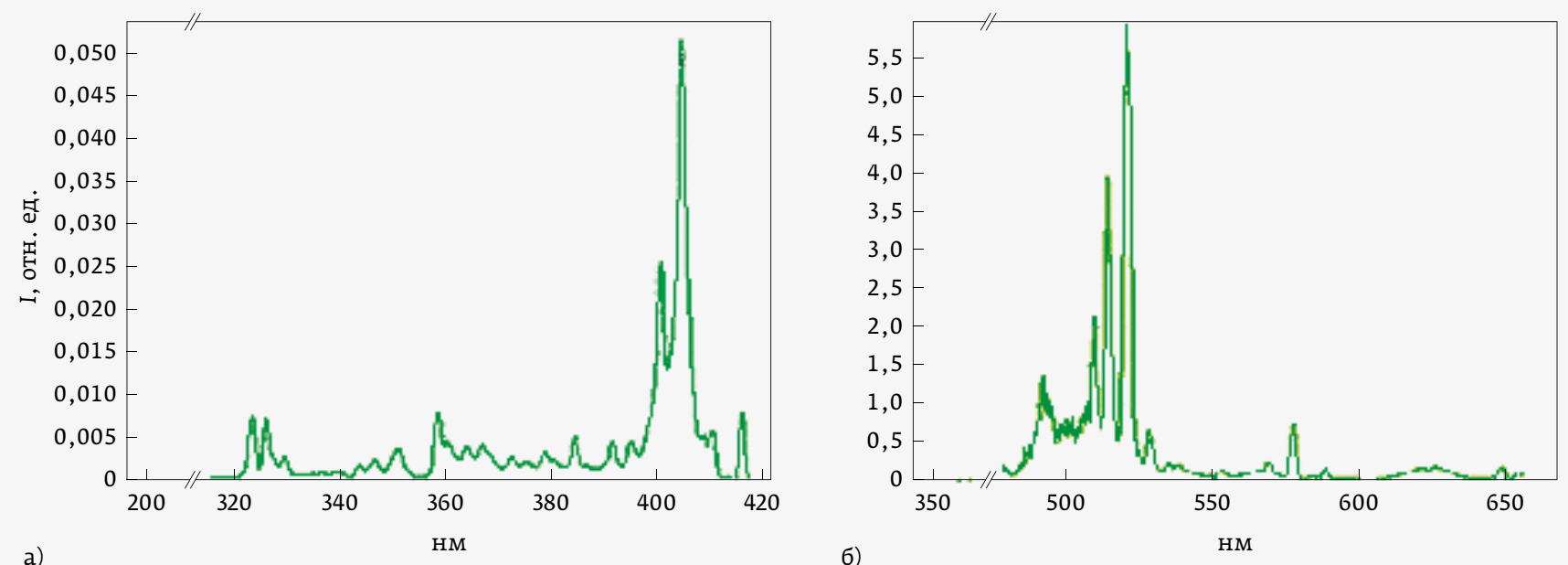

Рис. 4. Спектры меди в УФ- (а) и видимой (б) областях спектра (данные спектрометра) 


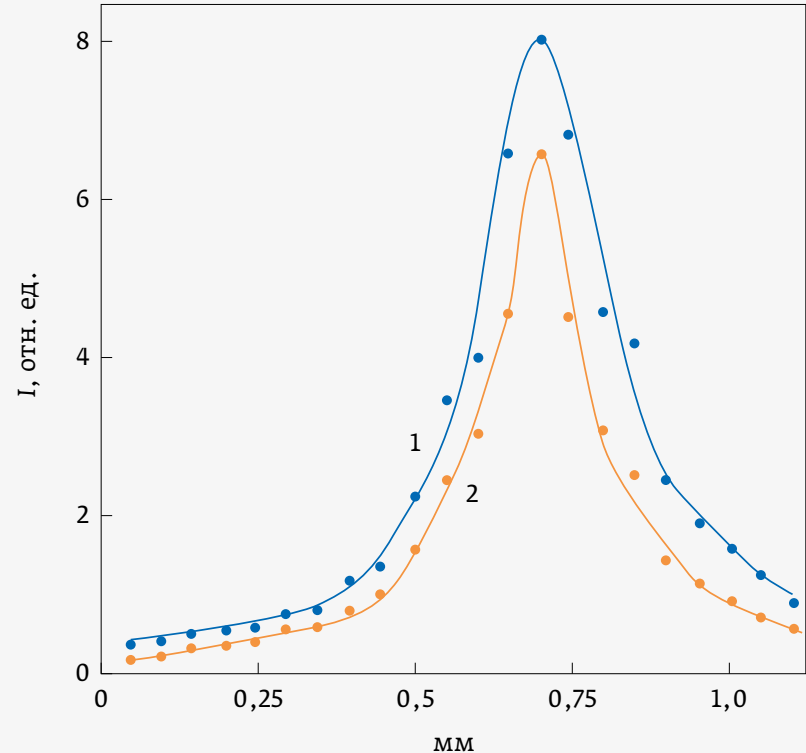

a)

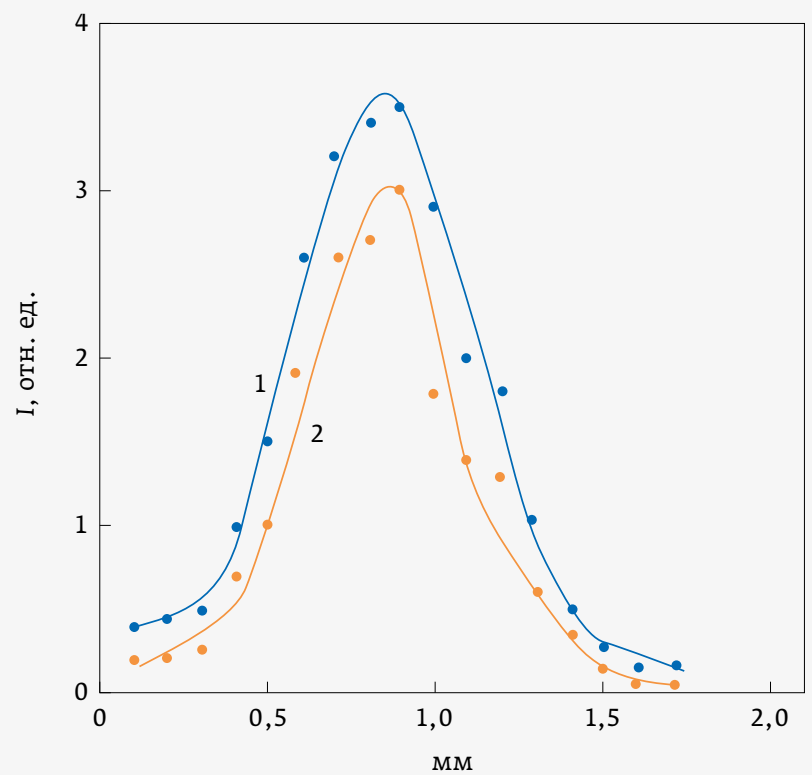

б)

Рис. 5. Интенсивность спектральных линий меди при перемещении горизонтальной диафрагмы (от поверхности мишени вверх) (а) и при перемещении вертикальной диафрагмы (слева направо) (б): 1 - 521,82 нм; 2 - 515,324 нм

миллиметра от поверхности мишени при атмосферном давлении. Из рис. 5 и 7 видно, что при сканировании плазменного факела вертикальной диафрагмой наблюдается практически полная симметрия интенсивностей спектральных линий относительно оси лазерного луча.

После замены щелевой диафрагмы на круглую диаметром 0,05 мм проведено исследование изменения интенсивности спектральных линий алюминия по оси лазерного луча. Эти данные представлены на рис. 8.
Максимальная интенсивность спектральных линий наблюдается на расстоянии порядка 0,25-0,3 мм от поверхности мишени.

Таким образом, показана возможность использования измерительного комплекса для исследования спектральных характеристик лазерного плазменного факела различных материалов мишеней. Использование и одновременно управление большим (до восьми) количеством спектрометров открывает новые перспективы в исследовании различных процессов, включая

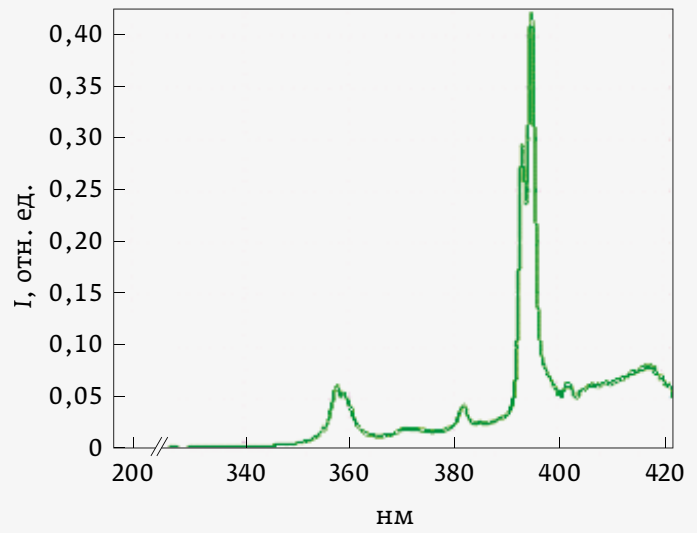

a)

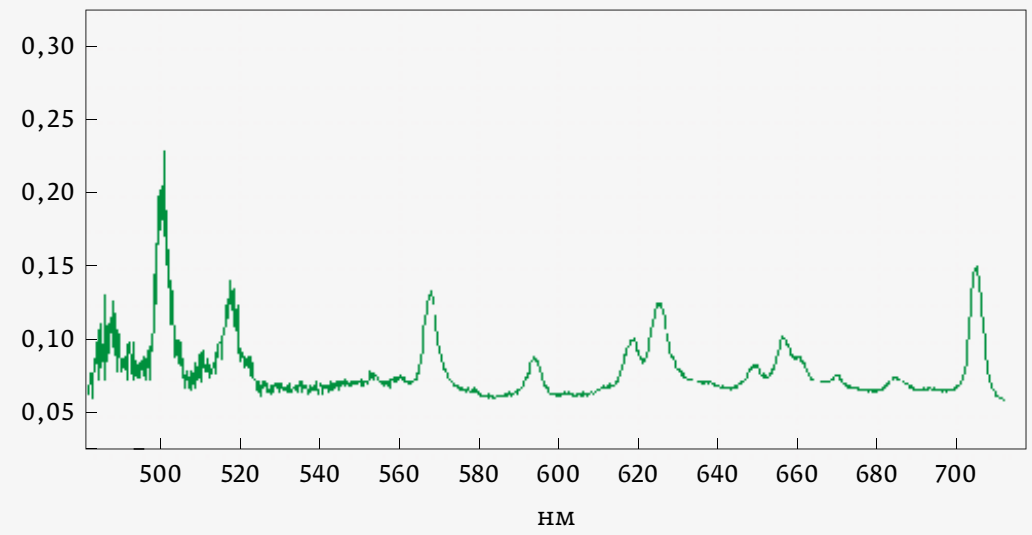

б)

Рис. 6. Спектры алюминия в УФ- (а) и видимой (б) областях спектра (данные спектрометра) 


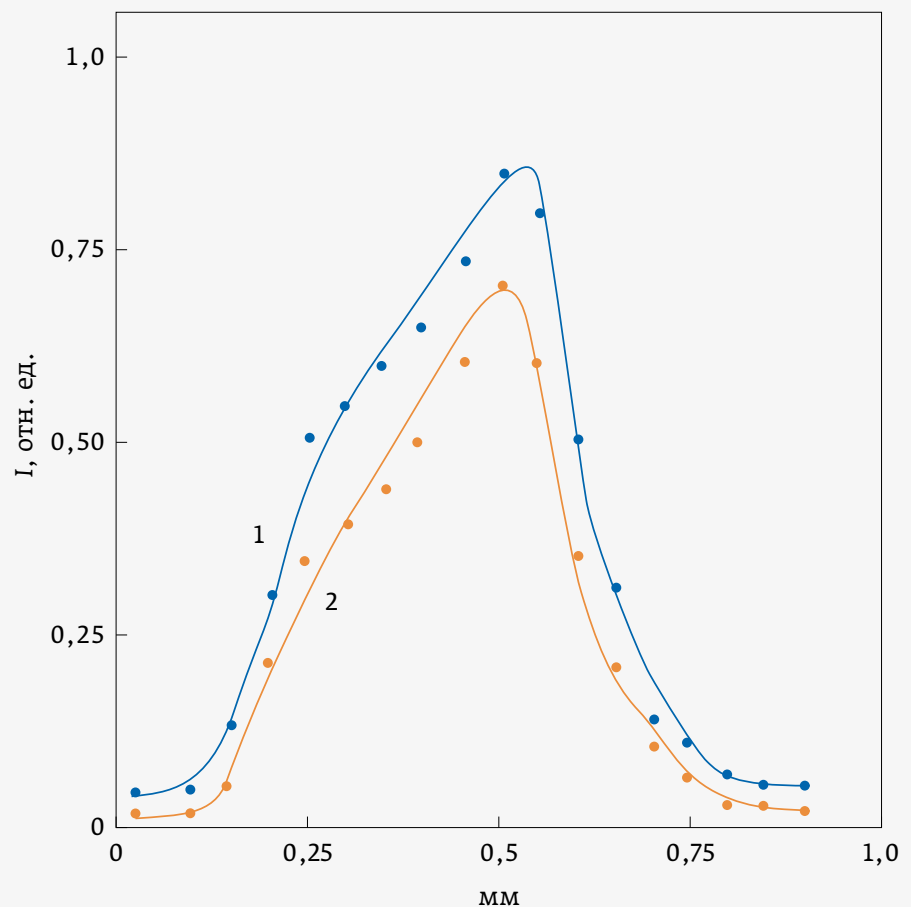

a)

Рис. 7. Интенсивность спектральных линий алюминия при перемещении горизонтальной диафрагмы (от поверхности мишени вверх) (а) и при перемещении вертикальной диафрагмы (слева направо) (б): 1 - 396,152 нм; 2 - 394,40 нм

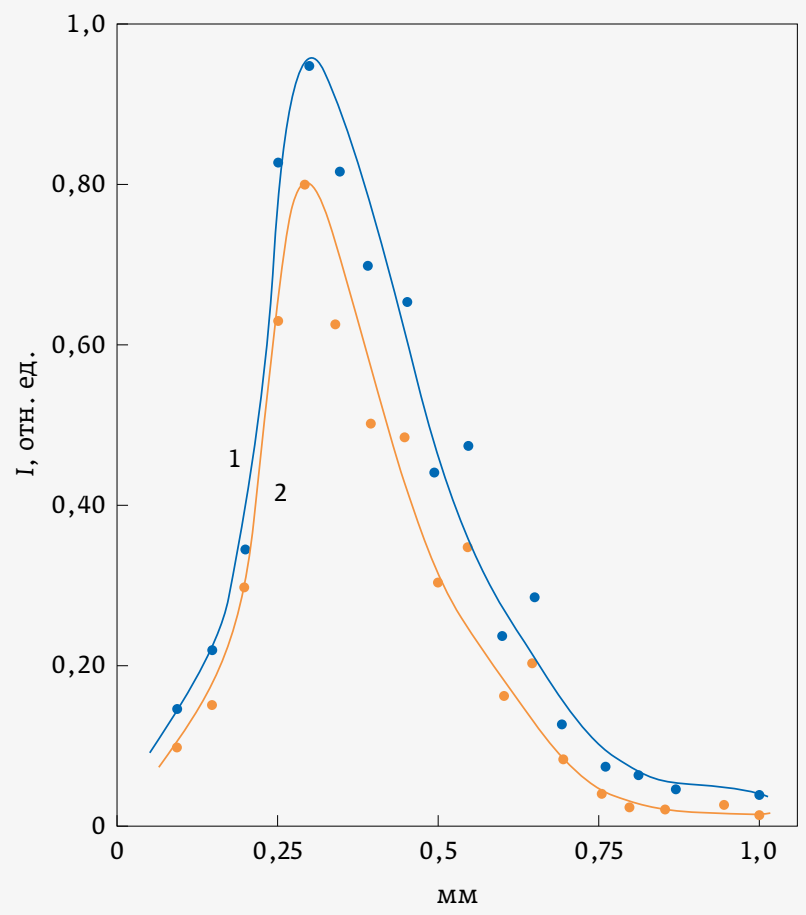

Рис. 8. Изменение интенсивности спектральных линий алюминия по оси лазерного факела (от поверхности мишени вверх): 1 - 396,152 нм; 2 - 394,40 нм

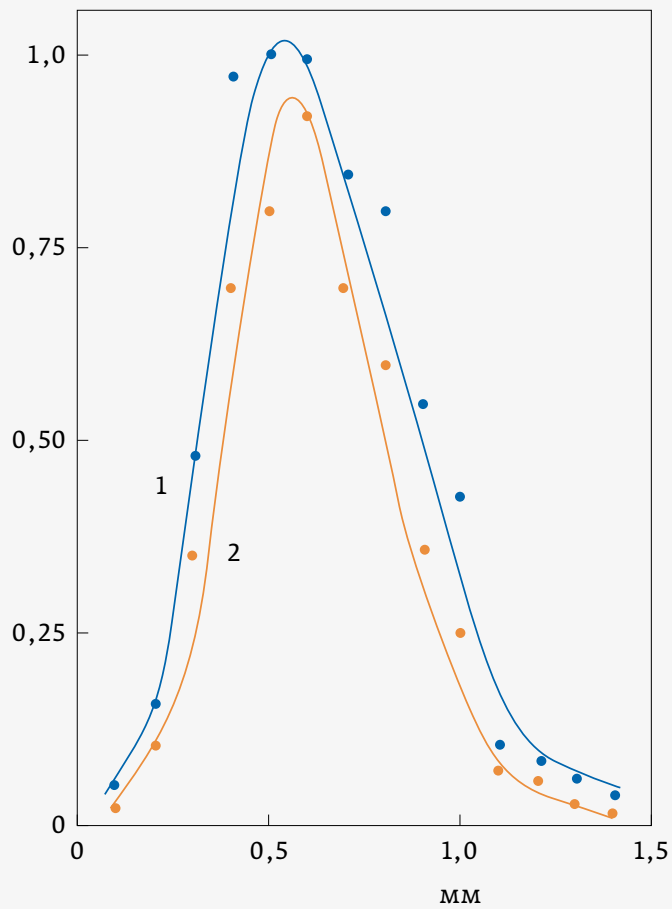

б)

быстропротекающие процессы и процессы с нестационарной температурой, связанных с измерением спектров излучения исследуемых объектов.

\section{СПИСОК ЛИТЕРАТУРЫ}

1. Тимченко Н.И. Программа для ЭВМ «Multi Spectral Pyrometry». Номер гос. регистрации 2019614365 от 03.04.2019.

2. Лапшинов Б. А., Суворинов А. В., Тимченко Н.И. Определение температуры излучающего объекта методом спектральной пирометрии // ЭЛЕКТРОНИКА: Наука, Технология, Бизнес. 2018. № 6 (177). С. 116-119.

3. Воробьев В.С. Плазма, возникающая при взаимодействии лазерного излучения с твердыми мишенями // Успехи физических наук. 1993. Т. 163. № 12. С. 51-83.

4. Лунин Л.С., Лунина М.Л., Девицкий О. В., Сысоев И. А. Импульсное лазерное напыление тонких пленок Al, Ga, GaP на подложках Si для фотопреобразователей // Физика и техника полупроводников. 2017. Т. 51. Вып. 3. С. 403-407.

5. Анисимов С. И., Имас Я.А., Романов Г.С., Ходыко Ю. В. Действие излучения большой мощности на металлы. - М.: Наука, 1970.

6. Стриганов А.Р., Свентицкий Н.С. Таблицы спектральных линий нейтральных и ионизованных атомов.М.: Атомиздат, 1966.899 С. 


\section{SSD от GS Nanotech}

\section{Первые российские}

твердотельные

накопители

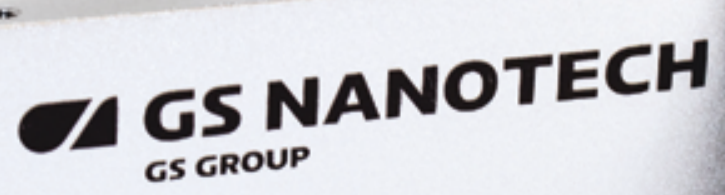

\section{Solid State Drive SATA $6 \mathrm{~Gb} / \mathrm{s} 2.5^{\prime \prime}$}

\section{Преимущества:}

[ Полный цикл производства в России

— Производство сертифицировано по ISO 9001:2015

" Максимальная 6езопасность данных: отсутствие षзакладок»

" Форм-фактор: 2,5" / М.2 / нестандартный по требованию заказчика

- Возможность 6рендирования продукта логотипом заказчика

- Диапазон рабочих температур: коммерческий $0 . . .+70^{\circ} \mathrm{C} /$ индустриальный $-40 . . .+85^{\circ} \mathrm{C}$

\section{Характеристики:}

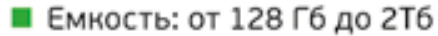

口 Интерфейс: SATA 3.06 Гбит/с; PCle Gen3×4 NVMe

- Тип памяти NAND Flash: MLC / 3D MLC / 3D TLC

- Мах скорость последовательной записи: до 520 M6/c (SATA) / до 3200 M6/c (PCle)

- Мах скорость последовательного чтения: до 560 M6/c (SATA) / до 3200 M6/c (PCle)

- Мах скорость произвольной записи: до 70000 IOPS (SATA / PCle)

[ Мах скорость произвольного чтения: до 90000 IOPS (SATA) / до 400000 IOPS (PCle)
Производство:

238050, Россия,

Калининградская область, г. Гусев,

ул. Индустриальная, А. 11 